\title{
LA TEORÍA DE LAS INTELIGENCIAS MÚLTIPLES EN LA ENSEÑANZA DE LAS LENGUAS
}

\author{
María Encarnación Carrillo García \\ Amando López López \\ Universidad de Murcia
}

\begin{abstract}
RESUMEN: En el siguiente trabajo analizamos la Teoría de las Inteligencias Múltiples de Howard Gardner enfocada en la enseñanza de las lenguas. Nuestro principal propósito es definirla, enumerar los tipos de inteligencias que contempla, así como describir las actividades que diferentes autores proponen en sus investigaciones para desarrollarla en el contexto de la enseñanza y aprendizaje de las lenguas.
\end{abstract}

PALABRAS CLAVE: Psicología de la Educación, lengua materna, lengua extranjera, enseñanza de idiomas, inteligencia.

\section{THE MULTIPLE INTELLIGENCE THEORY FOR THE TEACHING OF LANGUAGES}

ABSTRACT: In the following essay we analyse the Multiple Intelligence Theory of Howard Gardner focus on the teaching of languages, in order to describe its main points, such us: its description; the types of intelligences explained in it; and the activities, that some authors describe, for developing this theory in the teaching and learning language context.

KEYWORDS: Educational Psychology, mother tongue, foreign languages, language instruction, Intelligence.

Recibido: 30/11/2012

Aceptado: 15/08/2013 


\section{INTRODUCCIÓN}

El 11 de mayo de 2011, el psicólogo, investigador y profesor de la Universidad de Harvard Howard Gardner fue galardonado con el Premio Príncipe de Asturias de Ciencias Sociales. Dicho autor ha centrado principalmente su trabajo en el análisis de las capacidades cognitivas del ser humano, a partir del cual ha desarrollado su Teoría de las Inteligencias Múltiples. En su obra dicho investigador defiende que no existe una única inteligencia sino que cada persona posee al menos ocho inteligencias: inteligencia lingüística, lógico-matemática, cinético-corporal, musical, espacial, naturalista, interpersonal e intrapersonal.

Entendemos que asumir la teoría de Gardner supone un cambio significativo en el modelo educativo, ya que implica la toma en consideración de las potencialidades innatas del alumnado, de manera que la enseñanza se convierta en un proceso de estimulación de cada una de las inteligencias de la persona.

Las acciones educativas que se están Ilevando a cabo a partir de la teoría de Gardner son variadas como por ejemplo Proyecto Zero', iniciado en 1967 por Nelson Goodman y presidido por Gardner, con el propósito de estudiar y mejorar el aprendizaje de las artes, ha ido ampliando su campo de acción a otras disciplinas. Abordándose en él evaluaciones basadas en el rendimiento, la educación para la comprensión, el uso de las inteligencias múltiples para lograr una pedagogía más personalizada y la calidad de los esfuerzos interdisciplinares en la educación.

Hasta la fecha, una de sus contribuciones más relevantes es el modelo de una "escuela inteligente", basada en el aprendizaje como una consecuencia del acto de pensar y el aprendizaje como comprensión profunda que involucre el uso flexible y activo del conocimiento. La mayor parte de este sistema ha sido implantado en las escuelas públicas de Estados Unidos, especialmente aquellas con población menos favorecida. Por otro lado, Gardner también participa en el Goodwork Project, programa destinado a mejorar la calidad y la autoestima de los profesionales tomando como referencia los factores de excelencia y ética.

En este artículo analizamos su Teoría de las Inteligencias Múltiples y su utilidad para la enseñanza-aprendizaje de las lenguas.

\section{La Teoría de las Inteligencias Múltiples}

Basándonos en Larsen-Freeman (2000) y Richards y Rogers (2001: 115) cuando nos referimos a las Inteligencias Múltiples estamos hablando de una filosofía educativa que centra su atención en el alumno o alumna, y que contempla varias dimensiones de la inteligencia que pueden ser trabajadas y desarrolladas en el aula. Esta teoría se basa en los trabajos de Howard Gardner (1983, 1993, 1999) de la Graduate School of Education de la Universidad de Harvard. De acuerdo con Gardner (1993) los tests tradicionales que miden la inteligencia se centran sobre todo en la medida de la lógica y el lenguaje, y dejan de lado otros aspectos importantes de la misma sin analizar.

1. http://www.premiosprincipe.es/howard-gardner-premio-principe-asturias.html [Última consulta: 25/11/2012]. 
Según el autor (Gardner, 1993) cada persona está dotada de una inteligencia formada a su vez, por una combinación de inteligencias múltiples que varían en grado y profundidad, pudiendo todas ellas pueden ser aumentadas con la práctica y el entrenamiento. Además, a partir de sus planteamientos acerca de la inteligencia, se reconocen las diferencias existentes entre el alumnado, debido a que cada uno responde a sus propios estilos de aprendizaje, preferencias o capacidad intelectual. Por ello, es importante destacar que la práctica pedagógica se beneficia si reconocemos las diferencias entre el alumnado, analizamos las particularidades de cada grupo de aula y acomodamos nuestra práctica como docentes a la realidad con la que tenemos que trabajar.

Queremos destacar que en los últimos años diversos enfoques metodológicos han centrado su atención en las diferencias existentes entre el alumnado y en la necesidad de tenerlas en cuenta para abordar un adecuado planteamiento pedagógico, entre ellos se encuentran: Individualized Intruction, Autonomous Learning, Learner Training y Learner Strategies.

\section{TIPOS DE INTELIGENCIAS}

A su vez, el Multiple Intelligences Model propuesto por Gardner (1993) es un modelo que describe ocho tipos de inteligencia, entre las que se encuentra la inteligencia lingüística, la misma está relacionada directamente con la enseñanza de las lenguas (Christison, 1997, 1998, 2001). Además de la inteligencia lingüística, que analizaremos más adelante, encontramos en su modelo de inteligencia los siguientes tipos según su teoría:

- Inteligencia Lingüística: la habilidad para utilizar el lenguaje de forma especial y creativa, y que tienen bien desarrollada personas que desempeñan trabajos como el de abogado, escritores, editores, intérpretes, etc.

- Inteligencia lógica y matemática: es la habilidad para pensar de una manera racional, a menudo encontrada en profesionales que se dedican a la medicina, ingeniería, programación, ciencia, etc.

- Inteligencia espacial: es la habilidad para formar imágenes y modelos mentales de realidades especiales y del mundo como tienen los arquitectos y arquitectas, decoradores y decoradoras, escultores y escultoras, pintores y pintoras, etc.

- Inteligencia musical: tener un buen oído para la música, como en músicos y compositores y compositoras...

- Inteligencia corporal y kinestética (kinesthetic): tener un cuerpo bien coordinado como podemos ver en deportistas, bailarines y bailarinas, actores y actrices...

- Inteligencia interpersonal: la que tienen las personas que trabajan en contacto con otras personas como pueden ser los vendedores y vendedoras, políticos y políticas, profesores y profesoras...

- Inteligencia intrapersonal: en esta destacan las personas que se conocen bien a sí mismas y que son capaces de aplicar su talento de manera exitosa, la tienen las personas bien equilibradas y que se manejan bien en los diversos planos de la vida. 
- Inteligencia naturalista: es la inteligencia para entender y organizar las pautas de la naturaleza.

Según esta concepción de la naturaleza, en los últimos tiempos un gran número de educadores, padres y madres... consideran importante tener en cuenta aquellos talentos que demuestran tener los estudiantes para proponerles actividades concretas que trabajen aquellas inteligencias y facultades en las que parecen destacar. Por otro lado, según Richards y Rogers (2001: 116) a partir del trabajo de Gardner se han desarrollado teorías que definen otros tipos de inteligencia, como la inteligencia emocional (Goleman: 1996, 1999), la inteligencia mecánica, y la inteligencia práctica, pero Gardner defiende tan sólo las ocho inteligencias definidas por él, debido a que están avaladas por las pertinentes investigaciones.

Autores como Reid (1997) y Christison (1998), con respecto a la enseñanza de las lenguas, han criticado que la teoría de Gardner no se basa en las teorías lingüísticas existentes, ni en ninguna otra teoría acerca del aprendizaje; aunque en contraposición Richards y Rogers (2001: 117) entienden que las teorías de Gardner definen el dominio de la lengua como una facultad central para las personas, por lo que se aborda en el terreno educativo de manera integrada con la música, la actividad física corporal, las relaciones interpersonales; no de una perspectiva exclusivamente lingüística, sino desde una perspectiva comunicativa.

En lo que se refiere a la enseñanza de las lenguas, como hemos visto anteriormente Gardner (1993) acuñó el término inteligencia lingüística, aunque la concepción global de la Teoría de las Inteligencias Múltiples concibe que la lengua es algo más que la concepción lingüística de la misma, ya que existen otros factores relacionados con ella como el ritmo, el tono, el volumen; además del aporte que realizan al dominio de la lengua las demás inteligencias que describe son inherentes al ser humano, y además están influidas por el plano de los sentidos, lo que conlleva a una concepción multi-sensorial de la misma.

En cuanto a los objetivos de aprendizaje, la Teoría de las Inteligencias Múltiples no realiza una descripción de los mismos, aunque centra su atención en el contexto de aula (setting) debido a que es el soporte donde se desarrollarán las experiencias de aprendizaje, y que darán cuenta de un óptimo ambiente de aula que posibilite el desarrollo de las diferentes inteligencias y la adquisición del aprendizaje, de esta manera el alumnado será el objetivo mismo de la práctica educativa, el cual será estimulado convenientemente para que se convierta en un aprendiz mejor y más optimista con respecto a la lengua que aprende y usa.

Richards y Rogers, suscriben además que no hay un programa de estudios específico desarrollado a partir de la teoría de Gardner (1993), pero podemos ver una propuesta de los pasos a seguir en el aula, es decir, una secuencia básica alternativa a los programas educativos "tradicionales" y que desarrolla Lazear (1991) en cuatro fases:

- Fase 1: Despertar la inteligencia: a través de experiencias multisensoriales, como pueden ser: tocar, oler, ver, etc.; de manera que el alumnado puede ser sensibilizado hacia las diferentes facetas y propiedades de los objetos y de los eventos que suceden en el mundo que los y las rodea. 
- Fase 2: Amplificar la inteligencia: el alumnado agranda y mejora su inteligencia mediante objetos elegidos por ellos y ellas mismas y mediante eventos relacionados con su propia experiencia, a partir del debate y la discusión con sus compañeros y compañeras de las propiedades de los objetos y de las experiencias de cada uno y una.

- Fase 3: Enseñar con y para la inteligencia: en esta fase la inteligencia se une al objetivo de la clase, esto es, a los diferentes aspectos del aprendizaje de la lengua. En la práctica esto se realiza mediante hojas de trabajo, proyectos en pequeños grupos y debate-discusión.

- Fase 4: Transferencia de la inteligencia: el alumnado refleja las experiencias de aprendizaje de las fases previas, y realiza propuestas y retos para desarrollar lo aprendido en el mundo "real" (fuera del aula).

Podemos ver otro autor, Nicholson-Nelson (1998: 73), que realiza una propuesta para el aula de las ocho inteligencias descritas por Gardner (1998), en lo concerniente a individualizar el aprendizaje, y que ejemplifica en la siguiente lista de proyectos:

- Multiple intelligence projects: se basan en una o más inteligencias y se diseñan para estimular determinados tipos de inteligencias.

- Currículum-based projects: se basan en determinados contenidos del currículo y en las inteligencias que sirven para desarrollarlos.

- Thematic-based projects: se desarrollan a partir de temas relacionados con el currículo pero se dividen y clasifican de acuerdo con las diferentes inteligencias.

- Resource-based projects: estos proyectos se diseñan para proporcionar al alumnado oportunidades para la investigación de un determinado tópico usando las inteligencias múltiples.

- Student-choice projects: este tipo de proyecto está diseñado por el alumnado y pretende el desarrollo de las diferentes inteligencias en particular.

Por otro lado, según Richards y Rogers (2001: 119) existe profesorado que prefiere organizar la clase en torno a un grupo de actividades en las que cada una se trabaje un tipo de inteligencia, y en las que el alumnado se va desplazando de una a otra para llevarlas a cabo. A continuación resumimos los diferentes tipos de modelos posibles a desarrollar en el aula de lenguas:

- Juego de resistencia o fuerza (play to strength): si se quiere que un atleta o músico (o algún estudiante con estos talentos) se convierta en un buen alumno o alumna de la clase de lengua hay que estructurar el material de aprendizaje en torno a sus facultades.

- Proporcionar variedad (variety is the spice): el docente debe proporcionar al alumnado una gran variedad de actividades que giren en torno a cada una de las ocho inteligencias descritas, para que el aprendizaje se convierta en interesante, vivo y efectivo.

- Elegir la herramienta de aprendizaje adecuada (pick a tool to suit the job): la lengua tiene una gran variedad de dimensiones, niveles y funciones; hay que 
tenerlas en cuenta para elegir la actividad adecuada para el desarrollo de las inteligencias múltiples.

- Desarrollo global e integral de todas las inteligencias (all sizes fit one): la Teoría de las Inteligencias Múltiples contribuye, en el ámbito educativo, al desarrollo integral de la persona.

- Conocimiento de otras culturas (Me and my people): como en la concepción actual de la enseñanza-aprendizaje de las lenguas se concibe el aprendizaje de la lengua de acuerdo con el contexto cultural donde se da, lo cual llevará al alumnado al conocimiento de los diferentes tipos de inteligencias valorados en las diferentes culturas diferentes de la suya, y que también puede llegar a desarrollar.

De acuerdo con lo anteriormente expuesto, podemos discernir que el profesorado que utilice esta teoría, debe concebir la enseñanza como un proceso en el que el alumnado desarrolla las ocho inteligencias descritas por Gardner (1993) a través del trabajo en el aula, Campbel (1997: 19) lo explica así: "is not prescriptive. Rather, it gives teachers a complex mental model from which to construct curriculum and improve themselves as educators"; además Christison (1997) alienta al profesorado a entender, dominar y comprometerse con la teoría, así como administrar y crear su propio modelo de enseñanza de inteligencias múltiples: "connect your life's experiences to your concept of Multiple Intelligences" (Christison, 1997: 7).

En esta línea y de acuerdo con Richards y Rogers (2001: 120) el profesorado se convierte en diseñador del currículo, de unidades, analista, buscador o inventor de actividades, y responsable de la orquestación de un ambiente rico en actividades multisensoriales dentro de la realidad de aula, con sus características propias en lo que se refiere a tiempo, espacio y material:

Teachers then become curriculum developers, lesson designers and analysis, activity finders or inventors, and most critically orchestators of a rich array of multisensory activities within the realistic constraints of time, space, and resources of the classroom (Richards y Rogers, 2001: 120)

De este modo y como Christison (1999) apunta, cada docente no es únicamente el encargado de enseñar lengua sino que es responsable del desarrollo global del alumnado en la medida que la realidad de la materia que imparte se lo permite. (Christison, 1999: 12). En el caso del alumnado, y respecto al papel que desempeña en el aula, decir que es alentado a que visualice sus propias metas en términos más amplios, de manera que le lleve a tomar un mayor control de sus propias inteligencias múltiples, y que desarrolle su propio perfil de las mismas. Desde este punto de vista, el alumnado queda emplazado para que realice un proceso de crecimiento personal, para lo cual el modelo educativo de las inteligencias múltiples que pretende una formación integral de la persona (whole person) le será de utilidad convirtiéndolos en mejores alumnos y alumnas en general.

En cuanto a la organización del aula, el planteamiento educativo de las inteligencias múltiples pretende sus objetivos a partir de las actividades multisensoriales, que ya mencionamos anteriormente; y el uso de material "auténtico" (realia), es decir, que proviene del mundo real. Una organización de actividades de acuerdo con las 
inteligencias que se pretende trabajar podemos encontrar en Christison (1997: 7-8) organizadas de la siguiente manera:

- Inteligencia lingüística: lecturas, discusiones en pequeños y grandes grupos, uso de libros, juegos de palabras, escuchar grabaciones en audio, crear y publicar periódicos o textos de escritura creativa, diálogos entre estudiantes, cuentos, debates, noticiarios, memorizaciones, usar procesadores de texto.

- Inteligencia lógica/matemática: demostraciones científicas, problemas de lógica, puzzles, pensamiento científico, presentación lógica-secuencia de un tema determinado, crear códigos, problemas, cálculos.

- Inteligencia espacial: cuadros, mapas, diagramas, vídeos, películas, diapositivas, dibujos y fotografías artísticas, cuentos imaginativos, organizadores gráficos, telescopios, microscopios, actividades visuales, visualización, fotografía, usar mapas mentales, pintar, realizar collage, ilusiones ópticas, dibujos.

- Inteligencia corporal/kinestésica: movimientos creativos, cocinar, role-plays, actividades de comunicación-transmisión, estudio de campo, viaje de estudios, mímica.

- Inteligencia musical: escuchar música grabada, escuchar música en directo, realización de instrumentos por parte del alumnado, cantar, cantar en grupo, apreciar el humor que produce la música, Jazz Chants.

- Inteligencia interpersonal: trabajo en grupos cooperativos, tormenta de ideas en grupo, mediación de conflictos, juegos de mesa, trabajo en grupo.

- Inteligencia intrapersonal: trabajo individual e independiente, proyectos individualizados, opciones para realizar el trabajo de casa, inventarios o listas, entrevistas personales, auto-aprendizaje e instrucción programada, aprendizaje reflexivo, centros de interés.

Como ejemplo de trabajo en el aula, Christison (1997) describe los procedimientos a seguir en una clase de nivel inicial, y que gira en torno a la "descripción de objetos" organizada según las cuatro fases descritas anteriormente:

- Fase 1: Despertar la inteligencia: el docente trae numerosos objetos a la clase y el alumnado comprueba su textura, o si es comida su sabor. Este tipo de experiencias activa los componentes sensoriales de la persona lo que beneficia al desarrollo de las inteligencias.

- Fase 2: Amplificar la inteligencia: se le pide al alumnado que traiga objetos a la clase o que use algo de su posesión. El alumnado describirá en grupo las características del objeto atendiendo a lo percibido por los cinco sentidos. Esta información la registrarán en una hoja de trabajo.

- Fase 3: Enseñar con y para la inteligencia: en esta fase el profesorado refuerza y enfatiza las experiencias sensoriales del alumnado ayudando con el vocabulario relativo a ellas. El alumnado trabajará en grupo, por ejemplo completando una hoja de trabajo describiendo las características de los objetos.

- Fase 4: Transferencia de la inteligencia: en esta fase el alumnado reflexionará en grupo, completando tablas, etc.; acerca de la relación que tiene lo aprendido con la vida real y su aportación a la práctica. 
Con este ejemplo de trabajo de aula podemos comprobar que el alumnado desarrolla (Christison, 1997: 10-12): su inteligencia lingüística: describiendo objetos; su inteligencia lógica: determinando cuál es el objeto descrito; su inteligencia visual/espacial: describiendo cosas; su inteligencia interpersonal: trabajando en grupos; su inteligencia intrapersonal: reflejando la implicación personal en las tareas.

\section{Conclusiones}

Aprender una lengua no es solamente incidir en su gramática o estructuras sino que supone un importante ejercicio cognitivo además de afectivo-social. Este último motivo es una de las claves de esta aportación, ya que hemos pretendido ir más allá de ese punto de partida y reseñar la importancia de considerar a la persona en su globalidad. Poner en liza numerosas capacidades supone una aproximación más comprensiva a la adquisición de una lengua e implica un acercamiento al éxito en el proceso de hacerla propia.

Al tratar de enseñar una lengua, materna extranjera, el profesorado siempre se plantea la vinculación del proceso de enseñanza/aprendizaje con un paradigma lingüístico y sociocultural determinado. En el mismo, una de las claves estriba en los aspectos afectivos, entre los que se pueden incluir la autoestima, la inhibición o la motivación. Es evidente que los mismos están estrechamente relacionados con la apuntada Teoría de las Inteligencias Múltiples. Trabajos como el de Ferrándiz, Bermejo, Ferrando y Prieto (2006) inciden en los fundamentos psicopedagógicos de dicha teoría y sobre todo, en su transferencia al sector educativo donde principalmente se establece la enseñanza de lenguas. En este sentido, las propuestas prácticas de Barrios (2002), Antunes (2005) o Amstrong (2006) guían la actividad docente y plantean una vía de explotación de las inteligencias múltiples en la enseñanza en general y en la enseñanza de lenguas en particular.

Lógicamente, la Teoría de las Inteligencias Múltiples se encuentra unida a la concepción de los enfoques actuales en la enseñanza de lenguas. Al respecto, en la contribución de Lorenzo, Trujillo y Vez (2011) se vislumbra esa necesidad, ya que, por ejemplo, para el desarrollo de AICLE (Aprendizaje Integrado de Contenidos y Lenguas Extranjeras), es precisa la incorporación de las explicadas distintas inteligencias. La necesidad de utilizar variados recursos artísticos (Por ejemplo, Música o Literatura) o el uso de cuerpo, espacio o inteligencia lógica, supone una conexión directa entre enseñar una o varias lenguas y estimular las distintas inteligencias.

En el contexto educativo actual se pone de relevancia la relación entre las inteligencias múltiples y el desarrollo de las competencias básicas (Alart, 2010). Previamente Prieto y Ferrándiz (2001); Prieto y Ballester (2003) habían mostrado formas distintas de aproximarse a los aprendizajes, haciendo proliferar la estimulación de todas las inteligencias que se muestran en la persona. Todo ello confluye en un planteamiento de la Didáctica de la lengua y la literatura próximo al que postulan López Valero y Encabo (2013), donde el enfoque comunicativo prima y se trata de formar personas con unas habilidades que realmente sean operativas y funcionales. 
Por consiguiente, podemos concluir:

- Que tal y como nos indica Trujillo (2012), es necesaria una escuela mejor, sobre todo más entroncada con la vida de los estudiantes y la sociedad. Esto afecta a la enseñanza de lenguas y justifica la necesidad de integrar y desarrollar la Teoría de las Inteligencias Múltiples en el currículum.

- La concepción de persona como beneficiaría del aprendizaje es una de las claves de la transposición teórica de la Teoría de las Inteligencias Múltiples, ya que entender la diversidad y la necesidad de desarrollar un determinado potencial es crucial a la hora de plantear el proceso de enseñanza/aprendizaje de las lenguas.

- Por último, un mayor desarrollo de la teoría en las aulas y centros es necesario; además de advertir la aportación de nuevos textos, experiencias e investigaciones que relacionen la Teoría de las Inteligencias Múltiples con la enseñanza de lenguas.

\section{REFERENCIAS BIBLIOGRÁFICAS}

ALART, N. (2010). Una mirada a la educación desde las competencias básicas y las inteligencias múltiples. Aula de innovación educativa, 188, 61-65.

AMSTRONG, T. (2006). Inteligencias múltiples en el aula: guía práctica para educadores. Barcelona: Paidós.

ANTUNES, C. (2005). Juegos para estimular las inteligencias múltiples. Madrid: Narcea.

BARRIOS, Ma . E. (2002). Propuestas de aplicación de la Teoría de las Inteligencias Múltiples a la enseñanza de una lengua extranjera. Greta: revista para profesores de inglés, 10 (1), 25-29.

CAMPBEL, L. (1997). How teachers interpret MI Theory. Educational Leadership, 55 (1), 15-19.

CARRILLO GARCÍA, M. E. (2008). Roald Dahl y la Literatura Infantil y Juvenil en la Didáctica de las Lenguas. Una propuesta para la Mejora de la Competencia Literaria en el aula. Tesis doctoral. Murcia: Universidad de Murcia. Disponible en: http://www.tdx.cat/handle/10803/10763 [Fecha de acceso: 25/11/2012].

CHRISTISON, M. (1997). An introduction to multiple intelligence theory and second language learning. En J. Reid (ed.), Understanding Learning Styles in the Second Language Classroom. Englewood Cliffs, N.J.: Prentice Hall/Regents, pp. 1-14.

CHRISTISON, M. (1998). Applying multiple intelligence theory in pre-service and inservice TEFL education programs. English Language Teaching Forum, 36 (2), 2-13.

CHRISTISON, M. (1999). Multiple Intelligences: Teaching the whole student. ESL Magazine, 2 (5), 10-13.

CHRISTISON, M. (2001). Applying Multiple Intelligences Theory in the Second and Foreign Classroom. Burlingame: Alta Book Center.

FERRÁNDIZ, C.; BERMEJO, R.; FERRANDO, M. y PRIETO, M. D. (2006). Fundamentos psicopedagógicos de las inteligencias múltiples. Revista española de pedagogía, 64 (233), 5-19. 
GARDNER, H. (1983). Frames of mind: The Theory of Multiples Intelligences. New York: Basic Books.

GARDNER, H. (1987). La Teoría de las Inteligencias Múltiples. México: Fondo de Cultura.

GARDNER, H. (1993). Multiple Intelligences: The Theory and Practice. New York: Basic Books.

GARDNER, H. (1994). Educación artística y desarrollo humano. Barcelona: Paidós.

GARDNER, H. (1995). Inteligencias múltiples. La teoría en la práctica. Barcelona: Paidós.

GARDNER, H. (1995). Mentes creativas. Una anatomía de la creatividad vista a través de las vidas de S. Freud, A. Einstein, P. Picasso, I. Stravinsky, T. S. Elliot, M. Graham, M. Gandhi. Barcelona: Paidós.

GARDNER, H. (1996). La nueva ciencia de la mente. Historia de la revolución cognitiva. Barcelona: Paidós.

GARDNER, H. (1997). Arte, mente y cerebro. Una aproximación cognitiva a la creatividad. Barcelona: Paidós.

GARDNER, H. (1997). La mente no escolarizada. Cómo piensan los niños y cómo deberían enseñar las escuelas. Barcelona: Paidós.

GARDNER, H. (1998). Mentes líderes. Una anatomía del liderazgo. Barcelona: Paidós.

GARDNER, H. (1999). Who owns intelligence? The Athantic Monthly, February, 67-76.

GARDNER, H. (1999). Mentes extraordinarias. Cuatro retratos para descubrir nuestra propia excepcionalidad. Barcelona: Kairós.

GARDNER, H. (2001). El proyecto Sprectrum (I). Madrid: Morata.

GARDNER, H. (2001). El proyecto Sprectrum (II). Madrid: Morata.

GARDNER, H. (2001). El proyecto Sprectrum (III). Madrid: Morata.

GARDNER, H. (2002). Buen trabajo. Cuando ética y excelencia convergen. Barcelona: Paidós.

GARDNER, H. (2002). La educación de la mente y el conocimiento de las disciplinas. Lo que todos los estudiantes deberían comprender. Barcelona: Paidós.

GARDNER, H. (2002). La nueva ciencia de la mente. Historia de la revolución cognitiva. Barcelona: Paidós.

GARDNER, H. (2002). Mentes creativas. Una anatomía de la creatividad. Barcelona: Paidós.

GARDNER, H. (2003). La inteligencia reformulada. Las inteligencias múltiples en el siglo XXI. Barcelona: Paidós.

GARDNER, H. (2005). Las cinco mentes del futuro. Barcelona: Paidós.

GOLEMAN, D. (1996). Inteligencia emocional. Barcelona: Kairós.

GOLEMAN, D. (1999). La práctica de la inteligencia emocional. Barcelona: Kairós.

LARSEM-FREEMAN, D. (2000). Techniques and Principle in Language Teaching (Teaching Techniques in English as a Second Language). Oxford: Oxford University Press.

LAZEAR, D. (1991). Seven Ways of Teaching: The Artistry of Teaching with Multiple Intelligences. Palatine, III: IRI Skylight. 
LÓPEZ VALERO, A. y ENCABO, E. (2013). Fundamentos didácticos de la lengua y la literatura. Madrid: Síntesis.

LORENZO, L.; TRUJILLO, F. y VEZ, J. M. (2011). Educación bilingüe. Integración de contenidos y segundas lenguas. Madrid: Síntesis.

NICHOLSON-NELSON, K. (1998). Developing Students' Multiple Intelligence. New York: Scholastic.

PRIETO, M. D. y BALLESTER, P. (2003). Las inteligencias múltiples: diferentes formas de enseñar y aprender. Madrid: Pirámide.

PRIETO, M. D. y FERRÁNDIZ, C. (2001). Inteligencias múltiples y currículum escolar. Archidona (Málaga): Aljibe.

REID, J. (1997). Understanding Learning Styles in Second Language Classroom. Englewood Cliffs, N. J.: Prentice Hall/Regents.

RICHARDS, J. C. y ROGERS, S. T. (eds.) (2001). Approaches and Methods in Language Teaching. Cambridge: Cambridge University Press.

TRUJILLO, F. (2012). Propuestas para una escuela en el siglo XXI. Madrid: Catarata. 
